\title{
REDUCTION OF SURFACE ROUGHNESS BY MODIFICATION OF STEP-BUNCHED ALUMINUM NITRIDE LAYERS TOWARDS STEP- FLOW MORPHOLOGY
}

\author{
N. Tillner a, b, C. Brandl a , M.P. Hoffmann ${ }^{\text {a }}$, R. Moser ${ }^{\text {a }}$, A. Waag ${ }^{\text {b }}$, and H.-J. Lugauer ${ }^{\text {a }}$ \\ a OSRAM Opto Semiconductors GmbH, Leibnizstraße 4, 93055 Regensburg, Germany \\ ${ }^{\mathrm{b}}$ Institute of Semiconductor Technology, Technische Universität Braunschweig, Hans-Sommer-Straße 66, 38106 Braunschweig, Germany \\ Email: nadine.tillner@osram-os.com
}

Received 8 July 2019; accepted 30 September 2019

\begin{abstract}
Conventional aluminum nitride (AIN) template fabrication techniques, like hydride vapour phase epitaxy or AlN growth on patterned sapphire substrates, usually lead to step-bunched template surfaces. The resulting macrosteps cause emission broadening or even multiple-peak characteristics of ultraviolet light-emitting diodes (UV LEDs) fabricated on such AlN templates. In order to reduce these macrosteps and to provide a smoother surface, even without the need of a thick AlN deposition, a two-layer growth procedure is reported here. A three-dimensional (3D) - twodimensional (2D) sequential growth initiates a significant modification of the previous AlN surface morphology and simultaneously limits the evolving tensile strain. A primarily step-bunched surface with a surface roughness root mean square of $1.8 \mathrm{~nm}$ is successfully reduced by the two-layer growth procedure down to $0.8 \mathrm{~nm}$, without any film cracking. This distinct roughness reduction of more than $50 \%$ is achieved within an AlN thickness of only $1.3 \mu \mathrm{m}$. With a smoother surface, the electroluminescence characteristic of a UV LED structure is substantially improved. Instead of a double-peak emission, typical for LEDs grown on step-bunched templates, a single-peak emission and lower spectral width were achieved, indicating the high potential of the suggested two-layer technique for improving performance.
\end{abstract}

Keywords: MOVPE, AlN, step-bunching

\section{Introduction}

UV LEDs operating in the UV-C spectral range nowadays attract a considerable attention. They enable numerous applications ranging from gas sensing and curing to water purification and sterilization, at the same time being superior to commonly used mercury-based UV discharge lamps in terms of non-toxicity, compactness and robustness [1], 2]. To succeed in applications like water purification, UV-C LEDs require a high light output power, and thus a high internal and external quantum efficiency (IQE, EQE) [1]. The device efficiency correlates with the crystalline quality of the epitaxially grown films forming the UV LED [3]. Hence, suitable substrates or templates for the growth of aluminium gallium nitride (AlGaN)-based UV LEDs are indispensable for high device efficiency. High-quality Al-rich AlGaN layers grown on native substrates have been demonstrated, enabling a high IQE [4 5]. Since commercially available native AlN substrates for homoepitaxy are still rare, AlN templates on sapphire substrates generally serve as pseudo-substrates. Due to a high lattice mismatch and differences in thermal expansion coefficients, the growth of AlN on sapphire generically suffers from a high threading dislocation density (TDD) [2, 6 . As stated, the underlying template is crucial for the device performance; 
therefore, the crystalline quality of the AlN deposited on sapphire substrates has to be addressed [3]. Among various approaches for high-quality AlN grown on sapphire substrates, e.g. multilayer growth [2, \%, , 7 ] or high-temperature annealing of AlN films [8, 9], the deposition by hydride vapour phase epitaxy (HVPE) [10, 11] and the growth on nano-patterned sapphire substrates (nano-PSS) by metalorganic vapour phase epitaxy (MOVPE) 12-15 have been shown to be promising. However, the AlN surface morphology caused by the last two techniques is often defined by macrosteps [11, 13-15]. While their onset is still not fully understood, Bryan et al. stated that step-bunching is mainly a result of surface kinetics rather than of film strain state and arises due to high Al-adatom diffusion lengths [16-18]. The key parameter is the vapour phase supersaturation controlling the Al-adatom diffusion length. If the diffusion length exceeds the substrate terrace width, induced by a substrate miscut, step-bunching is enabled. Typical AlN growth parameters like high growth temperatures correspond to a low supersaturation, consequently leading to high $\mathrm{Al}$-adatom diffusion lengths and to macrostepping. Such a growth mode is characteristic of HVPE, as well as of AlN growth on nano-PSS by MOVPE due to high growth temperatures needed to favour coalescence.

Despite few reported advantages of step-bunching, like current micropaths in the subsequent cladding layers and carrier localization in the active region induced by a composition and thickness inhomogeneity in the AlGaN film [19, 20], the interface roughness of the basic AlN layer affects the overall UV LED performance by broadening the emission spectrum or even evolving a multiple-peak emission which is usually detrimental [18]. It is therefore desirable to transform AlN templates with macrosteps into step-flow morphology, yielding a flat surface and consequently sharp interfaces, and well-defined quantum well structures in the active region for a highly efficient UV LED device performance.

In this work, a route towards modification of macrostepped AlN surface morphology into stepflows is developed by taking into account the induced tensile strain state and potential cracking serving as a strain relaxation mechanism [21]. To modify the morphology and strain state, we pursued a two-layer AlN deposition with a 3D-2D sequence. The suitability of this technique is tested by evaluating the electroluminescence (EL) emission spectra of a subsequently grown $\mathrm{AlGaN}$-based heterostructure including multi-quantum wells in the UV-C emission range.

\section{Experiment}

The test structures were grown by MOVPE on 2 -inch $c$-plane sapphire substrates with a miscut of $0.2^{\circ}$ towards the $m$-plane. The standard precursors, trimethyl-aluminum (TMAl), trimethyl-gallium (TMGa) and ammonia $\left(\mathrm{NH}_{3}\right)$, with hydrogen $\left(\mathrm{H}_{2}\right)$ and nitrogen $\left(\mathrm{N}_{2}\right)$ as diluent gases, were used.

A template with an artificially created stepbunched surface was deposited by MOVPE serving as a model system for AlN deposition techniques, which usually exhibit such a characteristic morphology. The step-bunched AlN templates were obtained by a low temperature nucleation step followed by a high temperature growth $\left(>1250^{\circ} \mathrm{C}\right)$. These layers served as a basis for further experiments. The subsequent twolayer growth process on such step-bunched templates contained the deposition of an intermediate layer (IL, 3D growth) followed by second layer (SL) deposition (2D growth). The $300 \mathrm{~nm}$ thick IL was grown at a fixed V/III ratio of $\sim 2400$, a reactor pressure of 100 mbar and a TMAl molar flow rate of $140 \mu \mathrm{mol} \mathrm{min}{ }^{-1}$. The IL process temperature $\left(T_{\text {proc }}\right)$ was varied from 1000 to $1200^{\circ} \mathrm{C}$ in $50^{\circ} \mathrm{C}$ steps. We established the SL deposition, to promote step-flow growth of the AlN, by changing the $\mathrm{NH}_{3}$ flow rate compared to the IL, resulting in a V/III ratio of $\sim 38$. The SL thickness was kept constant at $1 \mu \mathrm{m}$, the process temperature at $1160^{\circ} \mathrm{C}$. The reactor pressure and the molar flow rate of TMAl remained unchanged for both layers. The subsequent AlGaN-based heterostructure consists of $200 \mathrm{~nm}$ undoped $\mathrm{Al}_{0.70} \mathrm{Ga}_{0.30} \mathrm{~N}$, $1300 \mathrm{~nm}$ n-type $\mathrm{Al}_{0.70} \mathrm{Ga}_{0.30} \mathrm{~N}: \mathrm{Si}$ and five pairs of $3 \mathrm{~nm} \mathrm{Al} \mathrm{Al}_{0.50} \mathrm{Ga}_{0.50} \mathrm{~N} / 11 \mathrm{~nm} \mathrm{Al}{ }_{0.63} \mathrm{Ga}_{0.37} \mathrm{~N}$ forming the multiquantum wells (MQWs). The MQWs are followed by $30 \mathrm{~nm}$ p-type AlGaN:Mg and $500 \mathrm{~nm}$ p-GaN:Mg.

The crystal and surface quality of the templates before and after the AlN MOVPE growth were investigated by X-ray diffraction, Nomarski differential interference contrast (DIC) and atomic force microscopy (AFM). Wafer-level EL studies were conducted to investigate the emission characteristics of subsequent grown MQW layers. 


\section{Results and discussion}

\subsection{The step-bunched AlN template}

Experiments were carried out on $\sim 3 \mu \mathrm{m}$ thick stepbunched AlN templates (Fig. 1), to serve as a model system for the characteristic step-bunched AlN that has to be transformed towards step-flow morphology. AFM measurements exhibited a roughness root mean square $(\mathrm{RMS})$ of $\mathrm{Rq}=1.8 \mathrm{~nm}$ with a mean step height of $5.6 \mathrm{~nm}$. The height ranged from 3.2 to $8.1 \mathrm{~nm}$. The threading dislocation density (TDD) of the AlN was $\sim 3 \cdot 10^{9} \mathrm{~cm}^{-2}$, determined by XRD measurements. The screw- and edge-type dislocation densities forming the TDD were closely related to the full width at the half maximum (FWHM) of XRD rocking curves in the (002) and (102) direction via the corresponding Burger vectors [20, 22].

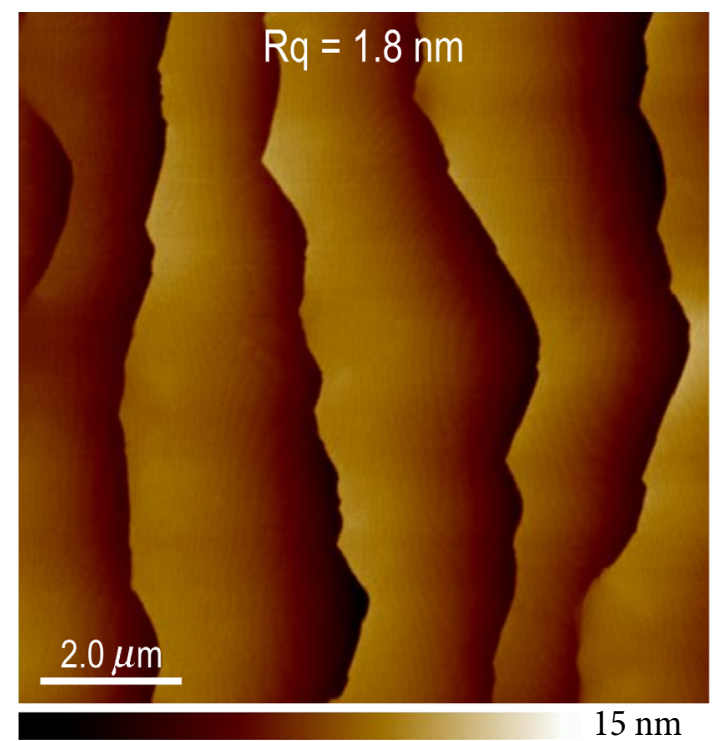

Fig. 1. AFM image with a $10 \times 10 \mu \mathrm{m}^{2}$ scanned area illustrating the step-bunched surface morphology of AlN templates. The roughness RMS (Rq) of $1.8 \mathrm{~nm}$ was determined with a mean step width of $2.4 \mu \mathrm{m}$ and a mean step height of $5.6 \mathrm{~nm}$.

\subsection{Two-layer overgrowth process on step-bunched templates}

On those step-bunched templates, the two-layer growth process, as described in the Experiment section, was carried out. The first layer of the twolayer deposition process, an interlayer (IL), was grown at process temperatures varying from 1000 to $1200^{\circ} \mathrm{C}$ in $50^{\circ} \mathrm{C}$ steps. On those varying interlayers, the second layer (SL) with constant growth parameters was deposited. Both layer morphologies, for the IL and the SL, were investigated by AFM in terms of morphology and surface roughness (Fig. 2). In Fig. 3, the temperature dependence on the roughness of IL and the entire stack is depicted.

The effect of IL deposition on the surface morphology is presented in Fig. 2(a-e). The samples in Fig. 2 $(a, b)$ indicate hexagonal V-pits with densities of $\sim 1 \cdot 10^{10}$ and $\sim 6 \cdot 10^{9} \mathrm{~cm}^{-2}$, respectively. This pit density (PD) is related to the growth temperature. The V-pit formation and their PD accordingly is assumed to be an effect of growth kinetics, controllable by, e.g. the growth temperature, and occurs at the existing threading dislocations of the underlying AlN layer, as shown for gallium nitride [23]. However, a discrepancy could be observed as the PD exceeds the TDD $\left(\sim 3 \cdot 10^{9} \mathrm{~cm}^{-2}\right)$ of the template in the case of the IL grown at $1000^{\circ} \mathrm{C}$ process temperature (Fig. 2(a)). This effect could be associated with the growth interruption between the template and IL growth, or to a strain relaxation mechanism supporting the V-pit formation [21]. In contrast to this V-pit morphology, the ILs illustrated in Fig. 2(c-e) exhibit 3D features with no significant morphology variance for growth temperatures between 1100 and $1200^{\circ} \mathrm{C}$. Generally two IL surface modifications of the previously step-bunched AlN film can be distinguished, a pitted surface and a 3D feature dominated surface. In Fig. 3, these two surface modifications can also be determined by their surface roughness. V-pit formation in ILs grown at temperatures $<1100^{\circ} \mathrm{C}$ produce a distinct surface roughening compared to the step-bunched template. The IL Rq is $5.9-10.7 \mathrm{~nm}$, significantly higher in contrast to the surface roughness for IL deposited at temperatures above $1100^{\circ} \mathrm{C}$, i.e. $1.4-1.8 \mathrm{~nm}$. At elevated temperatures $\left(\geq 1100^{\circ} \mathrm{C}\right)$, the surface roughness RMS is decreased compared to the primarily macrostepped AlN surface regardless of the obtained 3D features. For these samples, a gradual transition from the $3 \mathrm{D}$ to $2 \mathrm{D}$ growth mode is implicated. 3D features are obtained, while the surface roughness additionally is decreased indicating a trend to the $2 \mathrm{D}$ growth condition.

The corresponding SL surfaces covering the ILs are presented in Fig. $2(\mathrm{f}-\mathrm{j})$. For the SLs deposited on the ILs, grown at temperatures $\leq 1150^{\circ} \mathrm{C}$ (Fig. 2(f$\mathrm{h})$ ), the pre-arranged steps are modified towards 
(a)

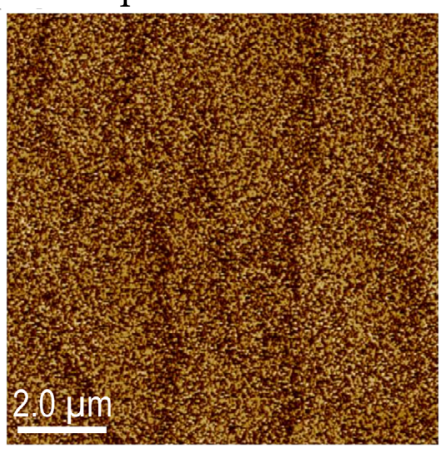

$60 \mathrm{~nm}$

(d)

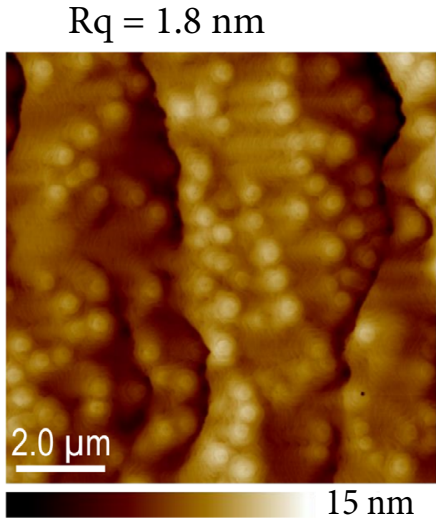

$\mathrm{Rq}=0.8 \mathrm{~nm}$

(g)

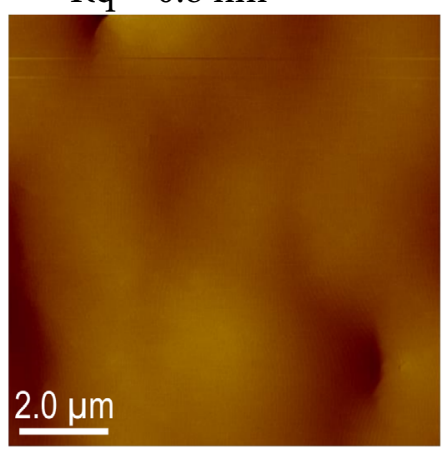

$15 \mathrm{~nm}$ (b)
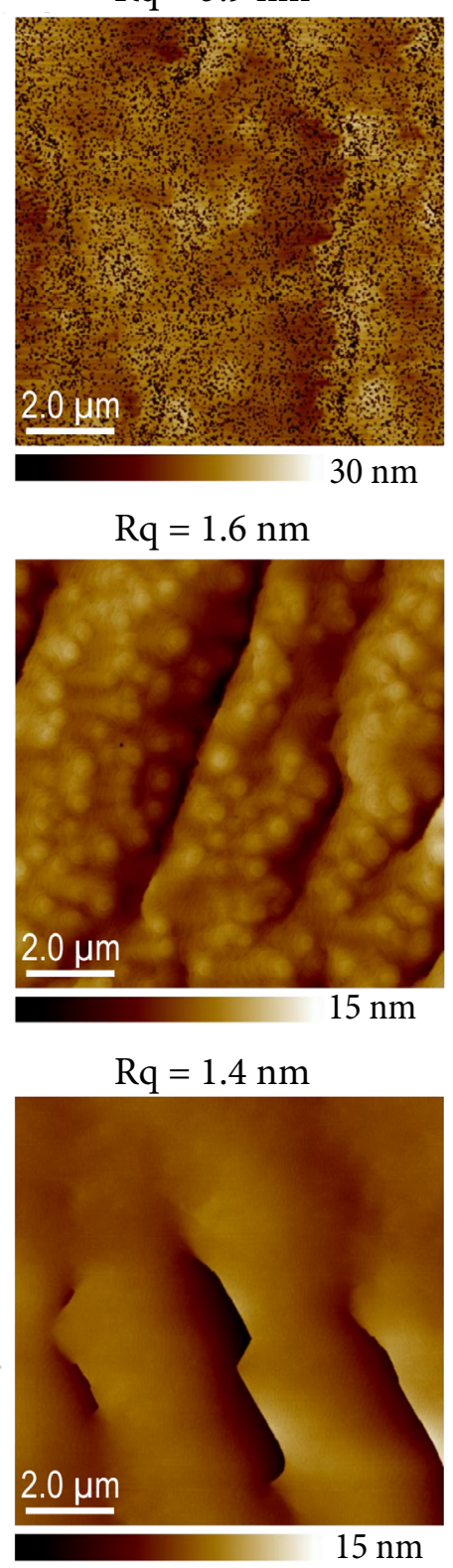

(h)

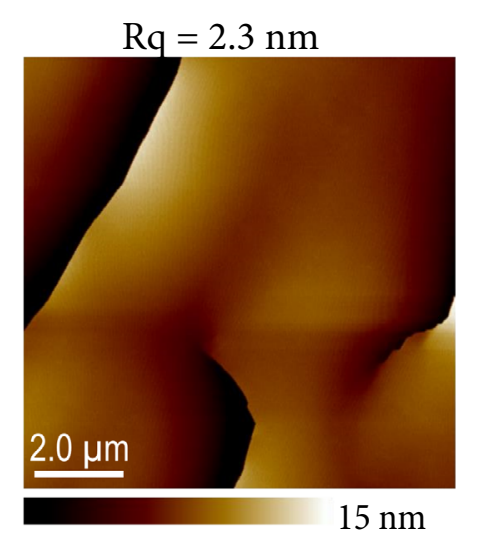

$15 \mathrm{~nm}$

\section{(j)}

(c)

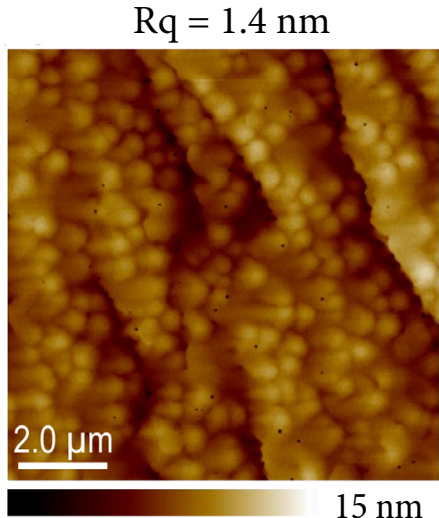

$15 \mathrm{~nm}$

(f)

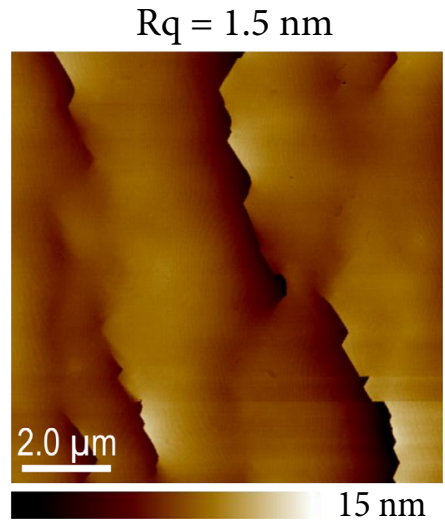

(i)

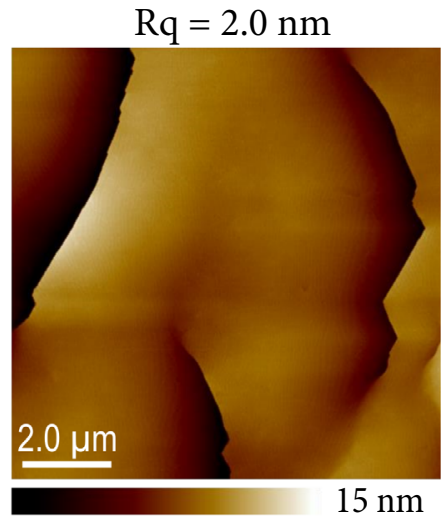

Fig. 2. $10 \times 10 \mu \mathrm{m}^{2}$ AFM images representing the surfaces of the two-step growth approach. The IL epitaxial growth (a-e) was carried out at five different process temperatures from $1000^{\circ} \mathrm{C}$ (a) to $1200^{\circ} \mathrm{C}$ (e) in $50^{\circ} \mathrm{C}$ steps. The surface after the overgrowth of SL is illustrated in $(\mathrm{f}-\mathrm{j})$. The remaining macrosteps resulted in step heights ranging from $3.5 \mathrm{~nm}(\mathrm{~g})$ to $13 \mathrm{~nm}(\mathrm{j})$. 


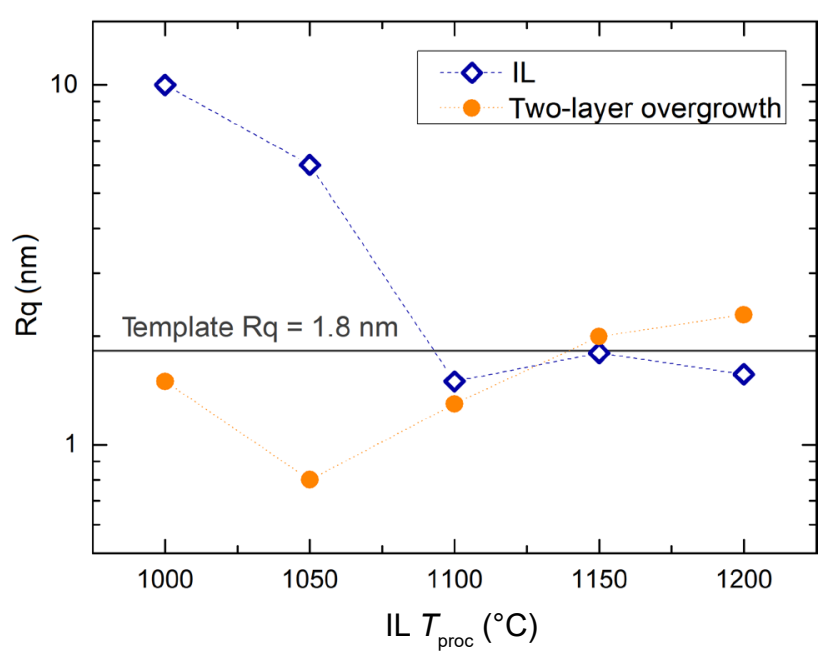

Fig. 3. Surface roughness RMS (Rq) of the IL (rhombs, blue online) and the overall two-layer growth (circles, orange online) on step-bunched templates with respect to the IL process temperature (IL $T_{\text {proc }}$ ).

smaller macrosteps. The interface presented in Fig. 2(g) shows no significant macrosteps reappearance and a sub-nm roughness. This indicates that the optimum for the IL growth is carried out at $1050^{\circ} \mathrm{C}$ process temperature (Fig. 2(b)), enabling a flat surface with bi-layer steps. In addition, the surface roughness of $0.8 \mathrm{~nm}$ of this AlN film illustrated in Fig. 3 represents the minimum of the curve. This observation confirms the suitability of the developed two-layer growth technique. When the IL growth temperature is increased further to 1150 and $1200^{\circ} \mathrm{C}$, macrosteps are partially extended up to a maximal height of $13.0 \mathrm{~nm}$ (Fig. 2( $(i, j))$.

The template and the following two-layer structure result in a total thickness of $\sim 4.3 \mu \mathrm{m}$. The strain state has therefore to be considered, since exceeding the tensile limit in the AlN film yields in a relaxation mechanism usually causing film cracking. Depositing solely the SL of the AlN two-step approach, with a similar total thickness of $\sim 1.3 \mu \mathrm{m}$ on a $\sim 3 \mu \mathrm{m}$ step-bunched AlN template, leads to cracking of the film (displayed in the DIC microscope image, Fig. 4(a)) with a roughness RMS of $1.3 \mathrm{~nm}$. These findings indicate that by directly starting with a $2 \mathrm{D}$ growth mode (that is indispensable for step flow morphology), mostly bi-layer stepping emerged on the larger-scaled macrosteps. However, the surface roughness of the macrostepped sample could not be significantly reduced. These findings also demonstrate that a pure SL growth condition favours film cracking before obtaining a smooth step-flowed surface. According to literature, the 3D mode IL should also lead to a limitation of tensile strain as shown for multilayer deposition with 3D-2D sequences [6, 月]. Employing a similar technique in this study, the sample with a pitted IL (Fig. 2(g), $3 \mathrm{D}$ growth) also indicates a successful strain management due to the observed crack-free surface (Fig. 4(b)). For IL process temperatures $\geq 1100^{\circ} \mathrm{C}$, a gradual transition in the IL from $3 \mathrm{D}$ to $2 \mathrm{D}$ growth is observed, leading to an increase in tensile strain and furthermore to sporadic cracks in the entire deposited stack (Fig. $4(\mathrm{c})$ ). In contrast, $3 \mathrm{D}$ layers deposited at temperatures below $1000^{\circ} \mathrm{C}$ do not support film-cracking; instead the coalescence of the considerably rough IL within an appropriate SL thickness is hindered.

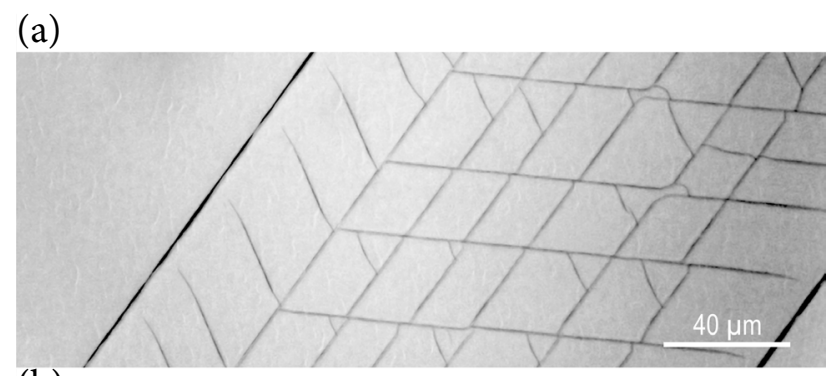

(b)

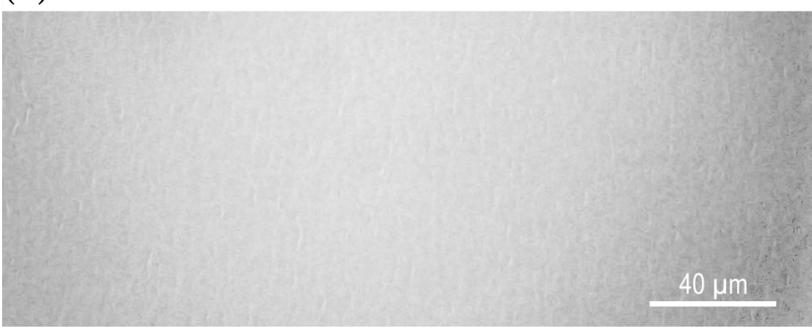

(c)

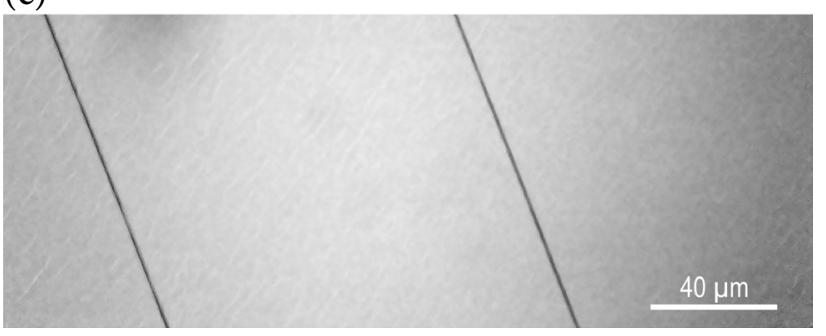

Fig. 4. 2D growth results in film-cracking caused by strain relaxation mechanisms (a), while a two-layer approach with an alternating 3D-2D growth avoids cracking (b). With elevated IL temperatures, the tensile strain in the film increases and sporadic cracking occurs (c). DIC microscope images were greyscaled and contrast enhanced for better visibility of the cracks. 


\subsection{EL spectra in dependence of different AlN template morphologies}

In order to investigate the effect of a smooth surface on LED performance, UV LED structures were grown on two AlN templates with distinctly different morphologies. To eliminate potential influences on the measurements caused by material quality deviations, both investigated epitaxial films were characterized in advance by XRD to ensure conformable and reasonable TDDs $\left(\sim 3 \cdot 10^{9} \mathrm{~cm}^{-2}\right)$. EL spectra of these heterostructures grown on a step-bunched template and a sub-nm rough template (achieved by the two-layer growth described here) are directly compared (Fig. 5). Both samples show a luminescence with the main MQW emission centered at $\sim 270 \mathrm{~nm}$. The MQW structure with an underlying step-bunched template exhibits a double-peak emission with a secondary peak centered at $294 \mathrm{~nm}$. This finding indicates spatial Ga-accumulations in the AlGaN cladding layers and the MQWs induced by the template's macrostepping [18]. In contrast, the MQW structure deposited on a smooth template, achieved by the two-layer overgrowth technique, shows a pure single-peak behaviour. Therefore, a homogeneous AlGaN composition is realized by the control of a low surface roughness of the template.

Additionally, not only a single-peak emission is enabled using a smooth basic AlN film, but also

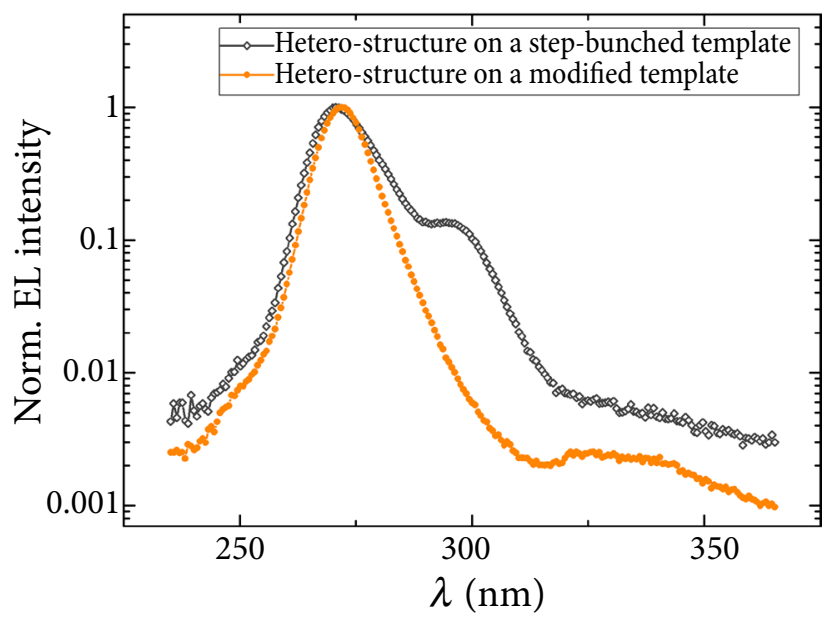

Fig. 5. Normalized EL intensity with respect to the wavelength $\lambda$ for AlGaN heterostructures grown on a step-bunched template (empty rhombs) and an atomically smooth AlN template (circles, orange online). Measurements were conducted at the wafer level. the EL spectral width (FWHM) of a MQW-structure deposited on the modified template is successfully narrowed from 13.5 to $10.1 \mathrm{~nm}$.

\section{Conclusions}

In summary, AlN templates with macrostepped surfaces were transformed towards step-flow morphology by a two-layer MOVPE deposition approach. The surface quality of the intermediate layer was studied at different process temperatures. This enabled an IL optimization in terms of successfully interrupting the existing underlying macrosteps structure. The reappearance of macrosteps is suppressed; simultaneously the evolving tensile strain was limited. Following the 3D submicrometre thick initial layer, the second stage serves as establishing the process for step-flow growth (reducing effectively the roughness). The RMS was decreased from 1.8 to $0.8 \mathrm{~nm}$, allowing for a significant reduction of $\sim 56 \%$ in the overall surface roughness at $1050^{\circ} \mathrm{C} \mathrm{IL}$ growth temperature.

The positive impact of the modified AlN surface on the AlGaN-based UV LED performance was assessed by EL emission spectra, showing a 25\% narrowed FWHM. By applying the developed technique to a step-bunched template, the MQW luminescence additionally is modified from a double-peak emission towards a single-peak characteristic. We thus demonstrated a successful surface modification technique of primarily step-bunched templates, usually achieved by high-quality AlN deposition techniques, like HVPE or growth on nano-PSS. Hence, a route towards an improved device operation is provided, as a narrow single-peak emission is required for many applications.

\section{Acknowledgements}

This work is partially supported by the German Federal Ministry of Education and Research (BMBF) within the 'Advanced UV for Life' Consortium. The authors thank Norbert Marschner for technical assistance with the MOVPE tool.

\section{References}

[1] M. Kneissl, T.Y. Seong, J. Han, and H. Amano, The emergence and prospects of deep-ultraviolet light-emitting diode technologies, Nat. Photonics 
13(4), 233-244 (2019), https://doi.org/10.1038/ s41566-019-0359-9

[2] H. Hirayama, N. Maeda, S. Fujikawa, S. Toyoda, and N. Kamata, Recent progress and future prospects of AlGaN-based high-efficiency deep-ultraviolet light-emitting diodes, Jpn. J. Appl. Phys. 53(10), 100209 (2014), https://doi.org/10.7567 JJAP.53.100209

[3] K. Ban, J.I. Yamamoto, K. Takeda, K. Ide, M. Iwaya, T. Takeuchi, S. Kamiyama, I. Akasaki, and H. Amano, Internal quantum efficiency of wholecomposition-range AlGaN multiquantum wells, Appl. Phys. Express 4(5), 052101 (2011), https:// doi.org/10.1143/APEX.4.052101

[4] Z. Ren, Q. Sun, S.Y. Kwon, J. Han, K. Davitt, Y.K. Song, A.V. Nurmikko, W. Liu, J. Smart, and L. Schowalter, AlGaN deep ultraviolet LEDs on bulk AlN substrates, Phys. Status Solidi C 4(7), 2482-2485 (2007), https://doi.org/10.1002 pssc.200674758

[5] Z. Bryan, I. Bryan, J. Xie, S. Mita, Z. Sitar, and R. Collazo, High internal quantum efficiency in AlGaN multiple quantum wells grown on bulk AlN substrates, Appl. Phys. Lett. 106(14), 142107 (2015), https://doi.org/10.1063/1.4917540

[6] N. Okada, N. Kato, S. Sato, T. Sumii, T. Nagai, N. Fujimoto, M. Imura, K. Balakrishnan, M. Iwaya, S. Kamiyama, et al., Growth of highquality and crack free AlN layers on sapphire substrate by multi-growth mode modification, J. Cryst. Growth 298, 349-353 (2007), https://doi. org/10.1016/j.jcrysgro.2006.10.123

[7] X. Zhang, F.J. Xu, J.M. Wang, C.G. He, L.S. Zhang, J. Huang, J.P. Cheng, Z.X. Qin, X.L. Yang, N. Tang, X.Q. Wang, and B. Shen, Epitaxial growth of AlN films on sapphire via a multilayer structure adopting a low- and high-temperature alternation technique, CrystEngComm 17(39), 7496-7499 (2015), https://doi.org/10.1039/C5CE01159K

[8] H. Miyake, G. Nishio, S. Suzuki, K. Hiramatsu, H. Fukuyama, J. Kaur, and N. Kuwano, Annealing of an AlN buffer layer in $\mathrm{N}_{2}-\mathrm{CO}$ for growth of a high-quality AlN film on sapphire, Appl. Phys. Express 9(2), 025501 (2016), https://doi. org/10.7567/APEX.9.025501
[9] S. Walde, S. Hagedorn, and M. Weyers, Impact of intermediate high temperature annealing on the properties of AlN/sapphire templates grown by metalorganic vapor phase epitaxy, Jp. J. Appl. Phys. 58(SC), SC1002 (2019), https://doi. org/10.7567/1347-4065/ab0cfd

[10]A. Claudel, V. Fellmann, I. Gélard, N. Coudurier, D. Sauvage, M. Balaji, E. Blanquet, R. Boichot, G. Beutier, S. Coindeau, et al., Influence of the V/ III ratio in the gas phase on thin epitaxial AlN layers grown on (0001) sapphire by high temperature hydride vapor phase epitaxy, Thin Solid Films 573, 140-147 (2014), https://doi. org/10.1016/j.tsf.2014.11.022

[11]T. Nomura, K. Okumura, H. Miyake, K. Hiramatsu, O. Eryu, and Y. Yamada, AlN homoepitaxial growth on sublimation-AlN substrate by low-pressure HVPE, J. Cryst. Growth 350(1), 69-71 (2012), https://doi.org/10.1016/j.jcrysgro.2011.12.025

[12]P. Dong, J. Yan, J. Wang, Y. Zhang, C. Geng, T. Wei, P. Cong, Y. Zhang, Y. Zeng, Y. Tian, et al., 282-nm AlGaN-based deep ultraviolet lightemitting diodes with improved performance on nano-patterned sapphire substrates, Appl. Phys. Lett. 102(24), 241113 (2013), https://doi. org/10.1063/1.4812237

[13]S. Hagedorn, A. Knauer, A. Mogilatenko, E. Richter, and M. Weyers, AlN growth on nano-patterned sapphire: A route for cost efficient pseudo substrates for deep UV LEDs, Phys. Status Solidi A 213(12), 3178-3185 (2016), https://doi. org/10.1002/pssa.201600218

[14]D. Lee, J.W. Lee, J. Jang, I.S. Shin, L. Jin, J.H. Park, J. Kim, J. Lee, H.S. Noh, Y.I. Kim, Y. Park, G.D. Lee, Y. Park, J.K. Kim, and E. Yoon, Improved performance of AlGaN-based deep ultraviolet lightemitting diodes with nano-patterned AlN/sapphire substrates, Appl. Phys. Lett. 110(19), 191103 (2017), https://doi.org/10.1063/1.4983283

[15]Z. Chen, J. Hoo, Y. Chen, V. Wang, and S. Guo, Study of AlN based materials grown on nanopatterned sapphire substrates for deep ultraviolet LED applications, Jpn. J. Appl. Phys. 58(SC), SC1007 (2019), https://doi.org/10.7567/13474065/ab09de 
[16]W.-K. Burton, N. Cabrera, and F.C. Frank, The growth of crystals and the equilibrium structure of their surfaces, Philos. Trans. Royal Soc. A 243(866), 299-358 (1951), https://doi. org/10.1098/rsta.1951.0006

[17]I. Bryan, Z. Bryan, S. Mita, A. Rice, J. Tweedie, R. Collazo, and Z. Sitar, Surface kinetics in AlN growth: A universal model for the control of surface morphology in III-nitrides, J. Cryst. Growth 438, 81-89 (2016), https://doi.org/10.1016/j. jcrysgro.2015.12.022

[18]I. Bryan, Z. Bryan, S. Mita, A. Rice, L. Hussey, C. Shelton, J. Tweedie, J.P. Maria, R. Collazo, and Z. Sitar, The role of surface kinetics on composition and quality of AlGaN, J. Cryst. Growth 451, 65-71 (2016), https://doi.org/10.1016/j. icrysgro.2016.06.055

[19] K. Kojima, Y. Nagasawa, A. Hirano, M. Ippommatsu, Y. Honda, H. Amano, I. Akasaki, and S.F. Chichibu, Carrier localization structure combined with current micropaths in AlGaN quantum wells grown on an AlN template with macrosteps, Appl. Phys. Lett. 114(1), 011102 (2019), https://doi.org/10.1063/1.5063735

[20]H. Xu, J. Jiang, M. Sheikhi, Z. Chen, J. Hoo, S. Guo, W. Guo, H. Sun, and J. Ye, Single peak deep ultraviolet emission and high internal quantum efficiency in AlGaN quantum wells grown on large miscut sapphire substrates, Superlattice Microstruct. 129, 20-27 (2019), https://doi. org/10.1016/j.spmi.2019.03.010

[21]I. Bryan, A. Rice, L. Hussey, Z. Bryan, M. Bobea, S. Mita, J. Xie, R. Kirste, R. Collazo, and Z. Sitar, Strain relaxation by pitting in AlN thin films deposited by metalorganic chemical vapor deposition, Appl. Phys. Lett. 102(6), 061602 (2013), https://doi.org/10.1063/1.4792694

[22]B.N. Pantha, R. Dahal, M.L. Nakarmi, N. Nepal, J. Li, J.Y. Lin, H. Jiang, Q. Paduano, and D. Weyburne, Correlation between optoelectronic and structural properties and epilayer thickness of AlN, Appl. Phys. Lett. 90(24), 241101 (2007), https://doi.org/10.1063/1.2747662

[23]S. Zhou, X. Liu, H. Yan, Y. Gao, H. Xu, J. Zhao, Z. Quan, C. Gui, and S. Liu, The effect of nanometre-scale $\mathrm{V}$-pits on electronic and optical properties and efficiency droop of GaN-based green light-emitting diodes, Sci. Rep. 8(1), 11053 (2018), https://doi.org/10.1038/s41598018-29440-4

\title{
PAVIRŠIAUS ŠIURKŠTUMO MAŽINIMAS KEIČIANT ALIUMINIO NITRIDO SLUOKSNIŲ LAIPTELIŲ SANKAUPAS TOLYGIU LAIPTELIŲ IŠSIDĖSTYMU
}

\author{
N. Tillner a, b, C. Brandl a , M.P. Hoffmann a ${ }^{\text {, }}$ R. Moser ${ }^{\text {a }}$, A. Waag ${ }^{\text {b }, ~ H .-J . ~ L u g a u e r ~}{ }^{\mathrm{a}}$ \\ a OSRAM Opto Semiconductors GmbH, Regensburgas, Vokietija \\ ${ }^{\mathrm{b}}$ Braunšveigo technikos universiteto Puslaidininkiu technologijos institutas, Braunšveigas, Vokietija
}

